\title{
El exilio republicano español en la Escuela Nacional de Economía de la UNAM: maestros y alumnos
}

\author{
Carlos Encinas Ferrer* \\ "Nada es más importante para el desarrollo de un país que una buena escuela de \\ economía (...) En el momento actual lo económico tiene una supremacía inevitable y \\ ha de ser resuelto en primer lugar. Una fuerte escuela de economía es absolutamente \\ necesaria en una nación".
}

John Maynard Keynes 1930 Residencia de Estudiantes ${ }^{1}$

Las diversas celebraciones que presenciamos en el año 2014 al cumplirse el 75 aniversario del inicio de la llegada del exilio republicano español a nuestro país, han dado lugar a un ejercicio de recuperación de memoria histórica, sobre las repercusiones que este hecho implicó para México. ¿Qué significó para los exiliados? Una nueva vida con libertad, una nueva patria para ellos y para sus hijos, una oportunidad más. De esto no hay duda. Pero ¿qué significó para México? Creo que nadie lo ha descrito mejor que Antonio Alatorre Chávez, el gran académico del Colegio de México: "La tarea que hicieron es de un valor absolutamente inapreciable, había que ver renglón por renglón qué ha sido México antes $y$ después de estos grandes hombres". ${ }^{2}$ Mucho se ha escrito sobre esto y no voy a detenerme en ello. Quiero resaltar algo muy específico: ¿Qué significó para la entonces Escuela Nacional de Economía (ENE) de la UNAM, hoy Facultad de Economía?

\footnotetext{
${ }^{1}$ Citado en http://www.aeca.es/viii_encuentro_trabajo_historia_contabilidad/ponencias/002.pdf

${ }^{2}$ Arturo García Hernández, "El crítico Antonio Alatorre subraya los invaluables aportes del exilio español” La Jornada, 1 de octubre de 2008. http://www.jornada.unam.mx/2008/10/01/index.php ?section $=$ cultura\&article $=\mathrm{a} 05 \mathrm{n} 1 \mathrm{cul}$.

* Carlos Encinas Ferrer nació en la Ciudad de México en el seno de una familia de republicanos españoles exiliados en 1939. Estudió la Licenciatura en Economía en la Escuela Nacional de Economía de la Universidad Nacional Autónoma de México (UNAM) y la Maestría en Educación en la Universidad Iberoamericana León. Obtuvo el Diploma en Estudios Avanzados (DEA) y Suficiencia Investigadora en Economía por la Universidad de Barcelona así como el Doctorado en Economía con calificación Sobresaliente "Cum Laude" por aquella Universidad.Académico de Tiempo adscrito al Centro de Economía Aplicada de la Escuela Nacional de Economía de la unAM de 1968 a 1969. Profesor Titular de la Facultad de Economía de la UNAM de 1966 a 1982. A partir de 2011 es profesor en la Escuela Nacional de Estudios Superiores de la UNAM en la ciudad de León, en el estado de Guanajuato. Fundó y dirigió las licenciaturas en Comercio Internacional y Economía en la hoy Universidad de León de 1992 a 1996. En la Universidad Iberoamericana León fue profesor de asignatura de 1995 a 1997, Académico de Tiempo en Licenciatura y Posgrado, así como Coordinador de la Licenciatura en Comercio Exterior y Aduanas de 1998 a 2001 y de 2003 a 2006. Director del Staff de Planeación y Evaluación de la Universidad Iberoamericana Puebla de enero de 2007 a diciembre de 2008. En agosto de 2009 fue nombrado Coordinador de la Maestría en Gestión Estratégica de Negocios Internacionales en la Universidad De La Salle Bajío, puesto que desempeña hasta la actualidad. A partir de septiembre de 2009 se integró como académico e investigador en la Universidad De La Salle Bajío y como profesor de asignatura en el Instituto Tecnológico de Estudios Superiores de Monterrey Campus León donde ha dado clases desde el año 1992. Ha sido profesor e investigador invitado en el posgrado de la Facultad de Economía y Empresa de la Universidad de Barcelona en 2013. Es miembro del Sistema Nacional de Investigadores (SNI) con el Nivel II.
} 


\section{La historia previa al exilio}

El 10 de febrero de 1929 se establece la sección de Economía dentro de la antigua Escuela Nacional de Jurisprudencia de la Universidad Nacional de México, aún no autónoma. Funcionó en el edificio de la Escuela de Jurisprudencia hasta 1934. En 1935, se convierte en la Escuela Nacional de Economía, y pasa a ocupar un edificio anexo a la Escuela de Jurisprudencia en el centro de la ciudad de México.

En los siguientes dos años la escuela tiene una vida errante en la que pasa de un edificio a otro hasta que en marzo de 1938, ocupa el edificio de la calle de Cuba núm. 92 donde permaneció hasta junio de 1954. Es precisamente en este edificio en donde se incorporarán como profesores los economistas del exilio y como alumnos algunos de sus hijos, también exiliados.

Es importante señalar que los maestros de economía del exilio provenían de un sistema en el cual existía la enseñanza de la economía como una rama de las escuelas y facultades de Derecho, tal y como había sucedido en México hasta 1935. Para ellos fue una novedad integrarse a un sistema universitario en el que la Economía contaba con una escuela dedicada únicamente a nuestra ciencia. Recordemos que no fue sino hasta 1943-1944 cuando se fundó en España la Facultad de Ciencias Económicas en la Universidad de Madrid y en 1954 la Facultat de Ciencies Economiques i Empresarials de la Universidad de Barcelona, mi segunda Alma Mater.

En 1954 la Escuela Nacional de Economía se traslada finalmente a la recién estrenada Ciudad Universitaria, cu como la llamamos coloquialmente, lugar donde estudiaremos los que pertenecemos a la llamada primera generación de nacidos en el exilio.

En el año de 1976, por acuerdo del Consejo Universitario, nuestra escuela se transformó en Facultad al tener ya una división de posgrado consolidada con el doctorado.

Antes de continuar debo señalar que la lista que a continuación presento no pretende ser exhaustiva y con toda seguridad hay omisiones, sobre todo entre los que fueron alumnos, tanto exiliados como entre los hijos del exilio. El orden en que los citó es arbitrario, no pretende presentarlos según su importancia y trascendencia.

\section{Los maestros del exilio}

A continuación me limito a presentar una breve semblanza y algunos recuerdos personales de los actores de nuestro relato. 
Antonio Sacristán Colás (Madrid, 1902; México, DF, 1986). Abogado, economista, político, empresario y académico nacido en España. Llegó a México, en 1939. Era Doctor en Derecho y catedrático de derecho mercantil por la Universidad de Madrid.

Desde 1939 fue profesor en la Escuela Nacional de Economía y en la Facultad de Derecho de la Universidad Nacional Autónoma de México (unAm). Formuló el proyecto que dio lugar a la Ley General de Instituciones de Crédito. En 1940 fundó la Sociedad Mexicana de Crédito Industrial siendo su Director General hasta 1963. También ocupó el puesto de Director General del Banco Mexicano, S. A. de 1958 a 1963. Fue Director General del Centro de Investigación y Docencia Económicas (CIDE) de 1977 a 1982. Profesor emérito de la Facultad de Economía de la unAm en 1985.

Javier Márquez Blasco, estudió Derecho en la Universidad Central de Madrid. Estudio el posgrado en la École Livre de Sciences Politiques de París y de 1934 a 1935 en la London School of Economics. A su llegada a México ejerció diversas actividades académicas en El Colegio de México, fue subdirector del Fondo de Cultura Económica (FCE) y codirector de la revista El Trimestre Económico. Durante sus años en el fCE tradujo numerosos títulos de economía. En esta misma época fue profesor de Economía en El Colegio de México y de Teoría del Comercio Internacional en la ENE, tema al que dedicó sus primeros trabajos académicos. En su obra, Inversiones internacionales de América Latina, realizó una crítica a la teoría clásica del comercio internacional y anticipó algunas de las ideas e instrumentos analíticos que servirían a Prebisch y los economistas de la Comisión Económica para América Latina (CEPAL) para explicar el subdesarrollo económico de la región y para elaborar su propio modelo de desarrollo. Al dejar el Fondo, en 1946, colaboró con el Banco de México. Fue funcionario del Fondo Monetario Internacional (FMI) en Washington de 1947 a 1951, y de la CEPAL en Santiago de Chile en 1951. Dirigió durante veinte años (1953-1973) el Centro de Estudios Monetarios Latinoamericanos (CEMLA), un centro de enseñanza e investigación creado en 1952 con el que las autoridades monetarias de Latinoamérica intercambiaron experiencias y buscaron mejorar el conocimiento de los aspectos monetarios de sus economías. Para todos los que éramos estudiantes entonces era obligado consultar su amplio acervo bibliográfico, la mayor parte de su propio Departamento Editorial. Sus análisis en el campo monetario tuvieron gran influencia en toda la región. Sus escritos más representativos los encontramos en su libro Problemas del oro, publicado en 1969 cuando el FMI estaba creando los Derechos Especiales de Giro. 
Ramón Ramírez Gómez (Madrid, 1913; México, 1972), maestro de primera enseñanza y presidente de la Federación Española de Trabajadores de la Enseñanza (FETE-UGT) en España, emigró a México en 1940, donde después de obtener el título de economista en la UNAm llegó a ser uno de sus más distinguidos catedráticos en los tiempos en los que la Escuela se abría tanto al pensamiento marxista como a la enseñanza de las teorías keynesianas. En 1951 obtuvo la cátedra de Teoría Monetaria y del Crédito en la Escuela Nacional de Economía. Ramírez centró su interés en estas materias y publicó diversas obras en las que defendió la tesis de que "los sistemas monetarios, más que instrumentos de desarrollo económico, son palancas en manos de los países poderosos para oprimir a los pueblos débiles, en cuyas economías tratan de influir a través de distintos métodos." En 1960 se integró al Instituto de Investigaciones Económicas (IIEc) de la unam, contribuyendo a la creación de su revista, Problemas del Desarrollo. Revista Latinoamericana de Economía. Sus explicaciones de clase, aunque fueron dándose a los alumnos en forma de apuntes a partir de 1958, adquirieron una forma impresa en 1963 cuando la Comisión Representante de los Alumnos del Grupo núm. 1, se encargó de su publicación. Estos apuntes alcanzaron una versión definitiva hasta poco después de su muerte, bajo el título de La moneda, el crédito y la banca a través de la concepción marxista y de las teorías subjetivas, de clara orientación marxista.

Su famosa conferencia celebrada el 7 de diciembre de 1960 en el Centro de Estudios Latinoamericanos de la Ciudad de México, bajo el título La Posible Revalorización del Oro y sus Efectos en la Economía de México, fue publicada en 1961 en forma de libro en la serie bimestral de Problemas Económicos de Nuestro Tiempo, de la revista Investigación Económica, con un magnífico prólogo de Alonso Aguilar Monteverde. Este pequeño libro tuvo un impacto muy grande en muchos de los que en la ENE estudiábamos, marcando, como en mi caso, el posterior interés en los temas monetarios.

Alfredo Lagunilla Iñárritu (Vizcaya, 1896; México, 1979) estudió Derecho y profesorado mercantil en España. Obtuvo el título de diplomado en el Instituto de Economía Aplicada de Bruselas. Trabajó en el Banco de España y publicó un interesante libro, Nuevos Ensayos sobre El Capital, una interpretación cooperativista de la economía clásica y de la economía marxista.

Su primer país de exilio fue la República Dominicana, pero al igual que muchos otros, no pudo resistir su clima ni las funciones productivas que el gobierno de aquel país había establecido para los exiliados republicanos y que no estaban dirigidas únicamente a ellos ya que se aplicaron a todo el que huía de la persecución política en Europa; recordemos entre muchos otros a Víctor Serge y su hijo, el pintor Vlady. 
De la República Dominicana se trasladó a México donde realizo diversos trabajos en entidades bancarias y financieras, llegando a ocupar el cargo de gerente del Departamento de Estudios Económicos de Valores del Banco de México y de profesor de Economía Monetaria y otras disciplinas económicas en el Instituto Tecnológico de México, posteriormente ITAM, y en la Escuela Nacional de Economía de la UNAm. Fue actor principal en la publicación de dos grandes revistas, Investigación Económica y El Trimestre Económico. Sus libros más destacados son Desarrollo y equilibrio de la economía mundial y Dinero y capital. En forma póstuma el Banco de México patrocinó la publicación de su gran obra, Historia de la banca y moneda en México.

Manuel Sánchez Sarto (Zaragoza, 1897; México, 1980) estudió las licenciaturas de Derecho y de Letras en la Universidad de Zaragoza. Entre 1921 y 1922 cursó estudios en las Universidades de Múnich, Kiel y Berlín. Fue director literario y gerente de la editorial Labor durante 10 años, de 1923 a 1939. Su labor como traductor fue de sin igual importancia en aquella editorial. Numerosas obras de política, sociología y economía fueron incorporadas a su catálogo, mostrando la amplia variedad de los intereses de Sánchez Sarto. Fue profesor de Historia Económica en la Facultad de Ciencias Jurídicas, Políticas, Económicas y Sociales de la Universidad Autónoma de Barcelona, proyecto abortado al fin de la Guerra Civil.

En 1939, se exilia, primero en París y Nueva York, y posteriormente en México. A su llegada a este país fue director gerente de la editorial Atlante (1939-1945), una de tantas fundadas por exiliados españoles. Trabajó también en el Fondo de Cultura Económica, para el que tradujo a Federico List, Richard Cantillon y Max Weber. Impartió la asignatura de Historia Económica en la Escuela Nacional de Economía de la UNAM, de la que llegó a ser catedrático en 1956. Fue investigador en el Instituto de Investigaciones Económicas y subdirector de la revista Investigación Económica, en la que publicó algunos de sus trabajos. Dirigió el Departamento de Economía del México City College.

Silva Herzog, lo invitó como asesor en la Dirección de Estudios Hacendarios de la Secretaría de Hacienda y Crédito Público de 1940 a 1945 y de 1949 a 1951. En el ínterin, 1946-1949, fue invitado por el presidente Rómulo Gallegos, a Venezuela, donde trabajó como economista asesor de la Corporación Venezolana de Fomento y como profesor de Economía y Sociología en las Facultades de Economía y Ciencias Jurídicas de la Universidad de Caracas. A su regreso a México fue asesor del Banco de México (1949-1953), al que llevó a otros exiliados españoles, como el geógrafo Leonardo Martínez Echevarría, el ingeniero Emilio Rodríguez Mata, quién fue destacado maestro en el Esime 
del Instituto Politécnico Nacional, y los economistas Alfredo Lagunilla y José Bullejos Sánchez. Fue economista asesor de la CEPAL en la subsede de México, manteniendo en ella importantes discrepancias teóricas y de política económica con Raúl Prebisch. De 1953 a 1956 fue profesor en la Escuela Superior de Administración Pública América Central (ESAPAC), creada por la CEPAL en Guatemala en 1957.

Faustino Ballvé Pallisé (Barcelona, 1887; México, 1958). Licenciado en Derecho en Barcelona, con estudios de economía en Berlín y en el Reino Unido. A su vuelta a Barcelona publicó acerca de algunos aspectos del socialismo y trabajó como traductor para diversas editoriales. Tuvo que exiliarse al fin de la guerra civil, pasando a Francia y posteriormente a México. En nuestro país revalidó su título de abogado, profesión que ejercería en su despacho profesional. Se dedicó a la docencia de la Economía: en la UnAm fue profesor de Economía en la Facultad de Derecho y en el Instituto Tecnológico de México. Lo he incorporado en nuestra lista pues aunque no tengo registro de que haya sido profesor de la ENE, si fueron sus textos libros de estudio obligado en muchas de nuestras asignaturas.

José María Urbano Guerrero (Granada, 1882; México, 1946), doctor en Derecho por la Universidad de Madrid, amplió estudios en las Universidades de Heidelberg y París, especializándose en Economía. Llegó a México en 1939, ejerciendo como profesor de Historia de las Doctrinas Económicas en la Escuela Nacional de Economía de UnAm hasta su muerte en 1946. Publicó artículos de economía en distintas revistas y tradujo el primer tomo de El apogeo del capitalismo de Werner Sombart para el Fondo de Cultura Económica.

\section{Los alumnos del exilio}

Rafael Izquierdo González (1928) estudió Economía en la ENE la UNAM. Durante unos años trabajó en la CEPAL y en los años de la década de los sesenta fue asesor del Secretario de Hacienda y Crédito Público y del presidente de México, formando parte del Grupo Secretaría de Hacienda-Banco de México. Fruto de esta experiencia fue su libro Política hacendaria del desarrollo estabilizador, 1958-1970. Después de dejar su trabajo en la Secretaría de Hacienda fue asesor de inversiones extranjeras del Presidente López Portillo y consejero del Banco de México. También fue economista de la Universidad de las Américas y de la Universidad McGill, de Canadá.

Trinidad Martínez Tarrago, cursó la licenciatura en Economía en la ENE (1951-1956) y el postgrado en la Universidad de Glasgow (Escocia). A su vuelta trabajó en la Secretaría de Hacienda y Crédito Público (1955-1959) y 
fue profesora adjunta de la asignatura Ciclos Económicos en la Escuela Nacional de Economía (1958). En 1963 vuelve otra vez al Reino Unido y al finalizar el curso 1970-1971 regresa a México, primero a la Universidad Anáhuac para reorganizar la Escuela de Economía. Al crearse el CIDE en 1974, dirigió su área de docencia y después el propio centro. En estos años, Martínez Tarrago trabajó con Fernando Fajnzylber en la redacción de Las empresas transnacionales, obra clásica en la literatura latinoamericana sobre empresas transnacionales. La llegada de Antonio Sacristán Colás a la presidencia del CIDE, con el que mantuvo unas difíciles relaciones, la llevaron a dejar esta institución en 1983.

Pedro Bosch García (Barcelona, 1922) llegó a México en 1941 con su padre, el historiador Pedro Bosch Gimpera, ex rector de la Universidad de Barcelona durante el Gobierno de la República Española. Nacionalizado mexicano, se licenció en la Escuela Nacional de Economía de la UNAm e hizo estudios de posgrado en la American University de Washington. A su vuelta organizó el Departamento de Economía del Mexico City College, fue profesor de Economía de la UNAM, economista de la Oficina de Investigaciones Industriales del Banco de México, miembro del consejo de administración de varias empresas públicas y miembro de la delegación oficial mexicana en las conferencias generales del Tratado de Montevideo iniciadas en 1986.

\section{Los hijos del exilio}

Emilio Sacristán Roy, Es hijo de Antonio Sacristán Colás. Graduado en Economía por la Universidad de Harvard, obtuvo la Maestría por la Universidad de Columbia. Comenzó su carrera docente en la Escuela Nacional de Economía de la UNAM como profesor adjunto del siempre recordado profesor Ricardo Torres Gaitán en la asignatura Comercio Internacional. Desde 1963 ha sido profesor de Macroeconomía en la ENE, actual Facultad de Economía. Desde 1988 es profesor de Finanzas en el Instituto Nacional de Administración Pública, en el que dirige el Programa de Alta Dirección de Entidades Públicas. Trabajó con su padre en el CIDE. Desde 1993 es miembro de la Academia Mexicana de Economía Política, de la que ha sido presidente.

José Andrés de Oteyza es Licenciado en Economía egresado de la ENE de la Universidad Nacional Autónoma de México, y tiene una Maestría en Economía en el King's College de la Universidad de Cambridge, fue designado Secretario de Patrimonio y Fomento Industrial por José López Portillo. Al terminar el gobierno de López Portillo fue nombrado por el presidente Miguel de la Madrid Embajador de México en Canadá de 1983 a 1987. 
Antonio Gazol Sánchez, hijo del jurista y economista Antonio Gazol Santafé, se licenció en Economía en la Escuela Nacional de Economía de la UNAM en 1966. Al terminar sus estudios fue Director del Instituto Mexicano del Café. Durante treinta y cinco años fue profesor de Economía y de Historia Económica en la Facultad de Economía de la UNAM. Ha publicado varios libros y ha escrito en revistas de divulgación económica y en prensa diaria. Ha sido presidente del Colegio Nacional de Economistas de México.

Carlos Roces Dorronsoro (México, 1945-2003), hijo de Wenceslao Roces, una de las figuras más destacadas del exilio republicano español. Licenciado en Economía en la Escuela de Economía de la UNAM, hizo un master en la London School of Economics y a su vuelta fue profesor de Economía en El Colegio de México durante casi treinta años hasta su muerte en 2003.

Matilde Souto Mantecón, nieta de José Ignacio Mantecón, ha sido profesora de la UNAM e investigadora en el Instituto de Investigaciones Dr. José María Luís Mora, es una de las más brillantes historiadoras económicas de México y cuenta ya con importantes publicaciones. Es miembro del Sistema Nacional de Investigadores en el Nivel I.

María Elena Cardero García. Estudió la licenciatura en Economía en la Escuela Nacional de Economía de 1961 a 1965. En la Secretaría de Relaciones Exteriores se desempeñó como Directora General de Relaciones Económicas Bilaterales con el Continente Americano y con América del Norte. Asimismo, fue miembro del equipo negociador del grupo de energía en el TLCAN. Es autora de varios libros y artículos que tratan sobre comercio internacional, políticas públicas y temas de género. Actualmente es profesora-investigadora de tiempo completo en la División de Posgrado de la Faculta de Economía de la UnAm. Ha compilado y editado varios libros sobre economía y equidad de género. Pertenece al Sistema Nacional de Investigadores en el Nivel II.

Consuelo Sáez Pueyo. Estudió la licenciatura en Economía en la Escuela Nacional de Economía de 1961 a 1965. Académica de nuestra escuela en los primeros años después de su titulación. Ha sido Directora General de Precios en la Secretaría de de Industria y Comercio, actual Secretaría de Economía, y posteriormente Directora General de Pesca.

Imanol Ordorika Sacristan. Imanol Ordorika nació en la Ciudad de México el 31 de octubre de 1958. Es hijo del destacado arquitecto Imanol Ordorika Bengoechea (Lekeitio, Bizkaia, 1931, México, D.F., 1988) y de María Josefa Sacristán (Madrid, España, 1936). Nieto del político y economista Antonio Sacristán Colás. Sus abuelos y sus padres llegaron a México como refugiados de la guerra civil española. Inmerso en el ambiente de una familia 
progresista, se formó en los ideales humanistas de la izquierda republicana española, de la Revolución Cubana, y del movimiento estudiantil mexicano de 1968. Ordorika obtuvo grados de maestría en educación (1993) y en sociología (1998) y el doctorado en ciencias sociales y educación (1999) en la Universidad de Stanford, Estados Unidos. En 1989 inició sus actividades como académico en el Instituto de Investigaciones Económicas (IIEc) de la UNAM. Desde 1999 ha sido integrante del Sistema Nacional de Investigadores en el que actualmente ocupa el nivel III. En 2006 fue distinguido con la Alfonso Reyes Chaire des Etudes Mexicaines de la Universidad de París III Sorbonne Nouvelle. Es miembro del Sistema Nacional de Investigadores en el Nivel III.

Jaime Ros Bosch. Hijo del arquitecto Jaume Ros y de Dolors Bosch Toldrá, exiliados en México al final de la guerra civil. Catedrático de la Facultad de Economía de la UNAM. Se ha desempeñado también como docente en la Universidad de Notre Dame y en el CIDE. Ha sido consultor de varios gobiernos latinoamericanos y organismos internacionales. Diploma en Economía, Universidad de Cambridge, Inglaterra, 1977-1978. Licenciado en Economía, UnAM, 1972-1974. B. A. en Ciencias Sociales, Universidad de Paris XII, 1969-1971 (beca del Gobierno de Francia). Senior Economist, Secretariado de la South Commission, Ginebra, Mayo 1988-Julio 1990. Profesor e Investigador en el CIDE, 1974-1987. Editor de "Economía Mexicana" en el CIDE, 1979-1985. Director del Departamento de Economía en el CIDE, Ciudad de México, 1978-1985. En la actualidad es Es miembro del Sistema Nacional de Investigadores en el Nivel III.

Fernando de Mateo y Venturini. Hijo del químico y economista exiliado, Eligio de Mateo Souza. Fernando estudió la licenciatura en Economía en la ENE. Recién egresado entro al servicio exterior mexicano donde es Embajador y Representante Permanente de México en la Organización Mundial del Comercio (OMC).

Recuerdo a muchos otros hijos del exilio egresados de la ENE y lamento no tener espacio para mencionarlos y describir sus aportaciones, tanto en la UNAM como en la vida de nuestro país. Tengo la esperanza que otros investigadores se interesen en ampliar este trabajo.

Nota: Una obra que me ayudo en mi trabajo y que nos proporciona información sobre este tema pero a nivel más amplio es Los Exiliados Españoles de la Guerra Civil en los Centros Superiores de Enseñanza de Economía de América Latina. ${ }^{3}$

\footnotetext{
${ }^{3}$ Manuel Martín Rodríguez, Los Exiliados Españoles de la Guerra Civil en los Centros Superiores de Enseñanza de Economía de América Latina, Revista Histórica de la Educación Latinoamericana, Redalyc. http://www.redalyc.org/articulo.oa?id=86918064009
} 